\title{
Immunotherapy: New Options in Gastrointestinal Cancers?
}

\author{
Andrea Tannapfel ${ }^{\mathrm{a}} \quad$ Anke Reinacher-Schick $^{\mathrm{b}} \quad$ Ulrich Hacker $^{\mathrm{c}} \quad$ Sylvie Lorenzen ${ }^{\mathrm{d}}$ \\ Markus Möhler ${ }^{e}$ Arndt Vogel ${ }^{f}$ \\ ${ }^{a}$ Institute of Pathology, Ruhr-University Bochum, Bochum, Germany; ${ }^{b}$ Department of Hematology, Oncology and \\ Palliative Care, Ruhr-University Bochum, Bochum, Germany; ${ }^{C}$ University Cancer Center Leipzig (UCCL), University

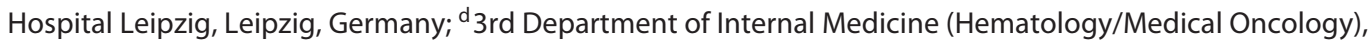 \\ Klinikum rechts der Isar der Technical University Munich, Munich, Germany; ${ }^{e}$ Department of Medicine I, University

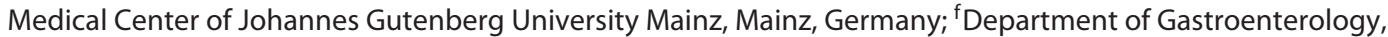 \\ Hepatology and Endocrinology, Hannover Medical School, Hannover, Germany
}

\section{Question 1: Considering present data in immunotherapy - which biomarkers are presently established in clinical practice in the treatment of GI cancers? Which biomarkers are upcoming?}

Hacker: Microsatellite instability-high (MSI-H) or mismatch repair deficiency (dMMR) represent the most important biomarkers based on a number of clinical trials showing that patients with MSI-H or dMMR tumors, including tumors of the gastrointestinal tract [1], significantly benefit from treatment with immune checkpoint inhibitors. Currently, PD-L1 expression (combined positivity score $\geq 1$ ) represents a prerequisite for the use of pembrolizumab in pretreated ( 2 or more lines) gastric and gastroesophageal junction (GEJ) cancer patients according to the approval by the FDA in the US [2]; however, such treatment has not yet been approved in Europe. Moreover, a combined positive score (CPS) of $>10$ was successfully applied in the Keynote 181 (press release Merck Nov. 14, 2018) and 180 [3] trials, in patients with both adenocarcinomas and squamous cell carcinomas (SCCs) of the esophagus for second- or third-line pembrolizumab treatment, respectively. Epstein-Barr virus

Chair: Andrea Tannapfel, Anke Reinacher-Schick. Participants: Ulrich Hacker, Sylvie Lorenzen, Markus Möhler, Arndt Vogel.
(EBV) positivity represents another very powerful biomarker in gastric cancer, as dramatic overall response rates up to $100 \%$ to pembrolizumab have recently been reported [4]. Finally, immunoscore analysis based on mRNA expression analysis of immune/interferon-related gene products holds promise to improve prediction of immune checkpoint therapy efficacy over MSI-H/dMMR in colorectal cancer (CRC) [5] and possibly other GI cancers. Finally, tumor mutational burden (TMB) is currently evaluated as a biomarker in GI cancers; however, data are controversial.

Lorenzen: Cancer immunotherapy is a promising new treatment option and is effective in a proportion of patients with gastroesophageal malignancies. However, biomarkers for selecting patients likely to benefit from immunotherapy in gastroesophageal cancer remain unproven. MSI and PD-L1 expression have been shown to predict a higher response to PD-1 inhibitors as highlighted by the recent approvals of pembrolizumab in treatment-refractory solid tumors with MSI status and the third-line or greater treatment of PD-L1-positive advanced gastric/GEJ cancers. However, PD-L1 still does not carry the highest sensitivity and specificity with variability in testing reported. Various phase II and III trials demonstrated that a PD-L1 expression of $\geq 1 \%$ in tumors

\section{KARGER}

(C) 2019 S. Karger AG, Basel
Prof. Dr. Anke Reinacher-Schick Department of Hematology, Oncology and Palliative Care Ruhr-University Bochum, Germany DE-44892 Bochum (Germany)

E-Mail Anke.Reinacher@rub.de 
is associated with an increased response rate; however, whether this is also correlated with a more favorable prognosis in terms of progression-free survival (PFS) and overall survival (OS) benefit is unclear. Another marker defining PD-L1 positivity is the CPS, where the number of PD-L1-positive tumor and immune cells (lymphocytes and macrophages) are divided by the total number of tumor cells evaluated and multiplied by 100 . Several studies with pembrolizumab could show that PD-L1 CPS-positive patients, specifically when the cutoff was defined as $\geq 10$, had an increased response rate und prolonged duration of response compared to patients with a CPS $<1$. MSI is present in a small but clinically relevant proportion of gastroesophageal cancers (approximately 4\%), and responses to PD-1 inhibitors appear to be more favorable in this subset from the small number of patients reported in the literature to date. Other predictive biomarkers for immunotherapy which are currently under evaluation are TMB, CTLA-4, FOXP3, LAG-3, as well as higher TIL infiltration.

Möhler: So far, MSI is the most attractive and best validated biomarker. Additionally, PDL1 by CPS scoring becomes another important predictive marker, particularly for advanced esophageal and gastric cancer. Herein, nearly all current phase 3 studies for first-line therapies included it at least as a co-primary endpoint for PFS or OS.

Vogel: First of all, I think we need to acknowledge that immunotherapy is not yet really established in GI cancers with the exception of rare MSI tumors, which is the best established biomarker. For patients with microsatellite stable (MSS) tumors, the most promising results have been reported in hepatocellular carcinoma (HCC) and gastric cancer. In HCC, there is so far no biomarker established to select patients for immunotherapy, but it appears that in up to $60-70 \%$, depending on the stage of the disease, tumor control can be achieved. Additionally, there are interesting data suggesting that combination therapies with tyrosine-kinase inhibitors and anti-angiogenic drugs might be more effective. In gastric cancer, there is increasing evidence that specifically patients with PD-1-positive tumors and patients with EBV-induced tumors derive the best benefit from immunotherapies.

\section{Question 2: Do you consider immunotherapy to become relevant in the near future for squamous cell cancer of the esophagus in line with data for head and neck cancers?}

Hacker: Current data are quite promising for PD-1 antibody treatment in this indication, based on the Keynote 181 (press release Merck Nov. 14, 2018) and 180 [3] stud- ies for second- or third-line pembrolizumab treatment, respectively, and I would assume that this treatment approach will become relevant in the future. Most data have so far been published for PD-1 targeting antibodies. However, studies of combinations with anti-CTLA-4 antibodies are also currently underway.

Lorenzen: Among histological types of esophageal cancers, SCCs are observed to have higher PD-L1 expression. According to The Cancer Genome Atlas for esophageal SCCs, SCCs resemble head and neck cancer more than esophageal adenocarcinomas. In the Keynote 180 trial presented by Shah et al. [3] at ASCO 2018, SCC did benefit more pronouncedly compared to adenocarcinoma of the esophagus. Currently, phase III trials with immunotherapy in combination with chemotherapy are recruiting both esophageal SCC and adenocarcinoma patients. Recently, the randomized phase 3 Keynote 181 trial, which randomized pembrolizumab versus chemotherapy in previously treated esophageal cancer, reported, in a recent press release, a significantly improved overall survival in patients with a PD-L1 expression of CPS $\geq 10$, independent of histology. Definitive data of this trial are awaited eagerly. Just recently, a press release on the 9th of January 2019 announced that the phase III ATTRACTION-3 trial (ONO-4538-24/CA209-473) evaluating nivolumab- versus taxane-based chemotherapy in patients with SCC of the esophagus, who were refractory to platinum first-line therapy, is positive, showing a significant extension of overall survival with nivolumab versus chemotherapy in PD-L1 unselected tumors. Dependent on the final results of both trials, it can be anticipated that immunotherapy might become a new treatment option in the future.

Möhler: Yes, after the positive publication of Keynote 181 and the press release for 473 pembrolizumab and nivolumab, these CPI will be addressed for approval in second-line therapy in Asia, the US, and Europe. As we don't have realistic options after platin/5FU-based firstline treatment, the positive results can be considered.

Vogel: Recently presented data from the Keynote 181 study indicate that patients with SCC of the esophagus can benefit from immunotherapies. Similar to gastric cancer patients with adenocarcinoma, a CPS of $>10$ might have prognostic relevance. Based on the published PFS and OS curves, there is still a need to find more effective combination therapies or predictive biomarkers.
Tannapfel et al. 
Question 3: What is your opinion on the role of immunotherapy for virus-associated cancers (e.g., EBV-associated gastric cancer and hepatitisassociated HCC)?

Hacker: Due to the extraordinarily high overall response rates (up to 100\%) reported so far for EBV-positive gastric cancer with pembrolizumab treatment, immune checkpoint therapy will definitely have a strong role in this indication [4]. Recovery of adaptive immunity has been demonstrated in models of viral hepatitis and HCC models during treatment with immune checkpoint inhibitors. The HBV-related HCC microenvironment, however, was demonstrated to be more immunosuppressive and exhausted than in nonviral-related HCC [6], pointing to the view that the role of immune checkpoint treatment in viral hepatitis-related HCC is by far less clear.

Lorenzen: The landmark analyses by The Cancer Genome Atlas proposed classifications based on comprehensive genomic profiling for 4 subtypes of gastric cancer: EBV infection, MSI, genomic stability, and chromosomal instability. Among these subtypes, the EBV-positive subtype and the MSI subtype seem to be inherently receptive to immune checkpoint blockade. EBV-positive gastric cancers seem to have the highest rates of PD-L1 expression in tumor and immune cells. On the other hand, among EBV-negative gastric cancers, only those with MSI seem to express PD-L1 within tumor cell. As recently shown by Kim et al. [4] in Nature Medicine, PD-1-targeted therapy was most effective in subgroups of patients with MSI-high, EBV-positive, or PD-L1(+) metastatic gastric cancer. Therefore, we now regularly test for both MSI and EBV and consider immunotherapy as a reasonable therapy option in these patients after failure of standard first-line therapy.

Möhler: For GI cancer, EBV-related carcinogenesis has been clearly correlated with immune and treatment responses to CPI. The recent publication in Nature Medicine in September 2018 [4] presented excellent data for the correlation to pembrolizumab. For hepatitis-associated HCC, this is not yet so clear.

Vogel: The role of underlying viral disease does not appear to play the same role in different GI cancers. In gastric cancer, several studies suggest that a higher efficacy can be seen in EBV-induced cancers. In contrast, a hepatitis B or $\mathrm{C}$ infection does not correlate with a higher efficacy in HCC. Overall, however, the data in HCC are premature, and we need to wait for more robust phase III data. There is so far no evidence in HCC that the anti-tumor immunotherapy has a lasting effect on the viral disease.

\section{Question 4: What is your present concept on immunotherapy of MSS CRC (e.g., induce immune response through chemotherapy or targeted agents)?}

Hacker: MSS CRC is found in around $95 \%$ of patients, and immunotherapy (i.e., immune checkpoint treatment) alone has no role in this group due to a lack of efficiency. Combinations of immunotherapy with either chemotherapy inducing immunogenic cell death (like oxaliplatin) or irradiation of target lesions to induce abscopal effects represent promising strategies to improve outcome and are currently tested in clinical trials. In addition, combinations of immune checkpoint inhibitors with the EGFR antibody cetuximab plus chemotherapy are promising due to the immune-modulatory effects of cetuximab by inducing NK cell-mediated cellular cytotoxicity [7]. Finally, a vast number of combination therapy approaches are currently under investigation [8].

Lorenzen: CRC can be divided into 4 different molecular subtypes, CMS1-4, with CMS1 types being characterized by MSI and strong immunogenicity. However, this classification is not implemented into clinical practice yet. Clinical application of $P D 1 / P D-L 1$-targeting checkpoint inhibitors in CRC has mainly focused on a subset of microsatellite instable (MSI-high) patients; however, this is only a small portion of patients (4\%). In future, TMB assessment might additionally accurately classify MSI tumors as TMB-high and may simultaneously identify a few additional CRC patients (approximately $3 \%$ ) as MSS/TMB-high. This subgroup may expand the population of CRC who may benefit from immune checkpoint inhibitor-based therapeutic approaches. At the moment, for the subset of immune-responsive MSI-high CRC patients, there are 3 FDA approvals: nivolumab and ipilimumab upfront according to the CheckMate-142 study, and nivolumab and pembrolizumab monotherapy after progression on a standard first-line therapy. However, there is no EMA approval.

However, for the majority of MSS CRC patients, treatment with immunotherapy is not an option. The combination of atezolizumab plus cobimetinib did not demonstrate statistically significant prolonged OS benefit versus regorafenib. So, unfortunately, for MSS CRC, immunemonotherapy is not an option, but there are a lot of ongoing efforts.

Möhler: Unfortunately, in many European countries, only MSI patients can be treated after failure of all available standard treatments with CPI. For MSS, it is even more difficult, as only few clinical data provide evidence for COI chemotherapy combinations. It is of interest that many companies are still cautious in doing such trials on 
their own. Thus, it is highly recommended to include patients into available studies from international or national groups, such as EORTC, AIO, FFCD, etc.

Vogel: The data on MSS CRC are so far very disappointing. There was very early evidence that the combination with chemotherapy does not meaningfully increase the activity of immunotherapy in MSS CRC. Subsequently, there were several very interesting approaches to turn cold tumors into hot tumors. There were specifically promising data for the combination with MEK inhibitors and anti-angiogenic agents. Unfortunately, the phase III data in MSS CRC with atezolizumab in combination with cobimetinib in the last-line setting and with Avastin as maintenance therapy were negative, clearly indicating that there are significant challenges to establish immunotherapy in MCC CRC. Interestingly, however, some of the approaches, such as the combination of immunotherapy with anti-angiogenic drugs, might work in other GI cancers. Last year, very promising data have been reported for the combination of atezolizumab with bevacizumab in HCC. Based on these data, the IMbrave150 phase III has rapidly finished recruitment, and the results are eagerly awaited.

\section{Question 5: Do you expect CAR-T cells to become relevant in GI cancers}

Hacker: The main problem related to CAR-T-cellbased treatment is its high toxicity, which is often related to off-target effects. Accordingly, a main prerequisite for the successful use of CAR-T-cell-based strategies in GI cancers is the identification of suitable targets [9]. Provided that such targets can be identified, there might be a role for CAR-T-cell-based therapy in some GI cancer types in the future.

Lorenzen: Compared to hematologic B-cell malignancies, genetically modified $\mathrm{T}$ cells expressing chimeric antigen receptors (CARs) therapy in solid tumors is just evolving. Also, in contrast to hematologic diseases, the full potential of CAR-T-cell therapy in solid tumors might be limited by the availability of cell surface antigens with sufficient cancer-specific expression. So, at the moment, there are only a few reports from early-phase CAR-T-cell clinical trials for solid tumors, showing promising activity. However, further exploration in solid tumors needs to be done. Maybe the combination of CAR-T-cell therapy with additional local and systemic therapies may enhance efficacy.

Möhler: This is still a highly experimental field. So far, we don't have enough effective antigens providing the basis for a broad use of this exciting strategy for other hematologic malignancies.

Vogel: The CAR-T-cell field is rapidly expanding, and extremely exciting data have been reported in liquid tumors, such as certain types of leukemia, lymphoma, and myelomas. The hurdles to implement CAR-T cells in solid tumors are clearly higher, most importantly the lack of specific targetable antigens. However, the encouraging results from early-phase trials have been only obtained in ErbB2-positive sarcomas. I believe that adoptive cell therapy could be one of the next, most important breakthrough strategies in the treatment of solid cancers.

\section{Participants}

Prof. Dr. med. Ulrich Hacker

University Cancer Center Leipzig (UCCL)

University Hospital Leipzig

Liebigstrasse 22, Haus 7, DE-04103 Leipzig (Germany)

E-Mail ulrich.hacker@medizin.uni-leipzig.de

Prof. Dr. med. Sylvie Lorenzen

3rd Department of Internal Medicine

(Hematology/Medical Oncology)

Klinikum rechts der Isar der Technischen

Universität München

Ismaninger Strasse 22, DE-81675 Munich (Germany)

E-Mail Sylvie.Lorenzen@mri.tum.de

Prof. Dr. med. Markus Möhler

Department of Medicine I, University Medical Center of Johannes Gutenberg University Mainz Langenbeckstrasse 1, DE-55131 Mainz (Germany)

E-Mail markus.moehler@unimedizin-mainz.de

Prof. Dr. Arndt Vogel

Department of Gastroenterology, Hepatology and Endocrinology, Hannover Medical School

Carl-Neuberg-Strasse 1, DE-30625 Hannover (Germany)

E-Mailvogel.arndt@mh-hannover.de 


\section{References}

1 Le DT, Durham JN, Smith KN, Wang H, Bartlett BR, Aulakh LK, et al. Mismatch repair deficiency predicts response of solid tumors to PD-1 blockade. Science. 2017 Jul;357(6349): 409-13.

2 Fashoyin-Aje L, Donoghue M, Chen H, He K, Veeraraghavan J, Goldberg KB, et al. FDA Approval Summary: Pembrolizumab for Recurrent Locally Advanced or Metastatic Gastric or Gastroesophageal Junction Adenocarcinoma Expressing PD-L1. Oncologist. 2019 Jan;24(1):103-9.

3 Shah MA, Kojima T, Enzinger PC, Hochhauser D, Raimbourg J, Hollebecque, et al. Pembrolizumab for patients with previously treated metastatic adenocarcinoma or squamous cell carcinoma of the esophagus: Phase 2 KEYNOTE-180 study. J Clin Oncol. 2018; 36(15 suppl):4049.
4 Kim ST, Cristescu R, Bass AJ, Kim KM, Odegaard JI, Kim K, et al. Comprehensive molecular characterization of clinical responses to PD-1 inhibition in metastatic gastric cancer. Nat Med. 2018 Sep;24(9):1449-58.

5 Mlecnik B, Bindea G, Angell HK, Maby P, Angelova $\mathrm{M}$, Tougeron $\mathrm{D}$, et al. Integrative Analyses of Colorectal Cancer Show Immunoscore Is a Stronger Predictor of Patient Survival Than Microsatellite Instability. Immunity. 2016 Mar;44(3):698-711.

6 Lim CJ, Lee YH, Pan L, Lai L, Chua C, Wasser $\mathrm{M}$, et al. Multidimensional analyses reveal distinct immune microenvironment in hepatitis $B$ virus-related hepatocellular carcinoma. Gut. 2018 Jul;gutjnl-2018-316510.
7 Stein A, Binder M, Al-Batran S-E, Hinke A, Waberer L, Goekkurt E, et al. Avelumab and cetuximab in combination with FOLFOX in patients with previously untreated metastatic colorectal cancer (MCRC): Results of the safety run-in phase of the phase II AVETUX trial (AIO-KRK-0216). J Clin Oncol. 2018;36(15 suppl):3561.

8 Tang J, Shalabi A, Hubbard-Lucey VM. Comprehensive analysis of the clinical immunooncology landscape. Ann Oncol. 2018 Jan; 29(1):84-91.

9 Townsend MH, Shrestha G, Robison RA, O'Neill KL. The expansion of targetable biomarkers for CAR T cell therapy. J Exp Clin Cancer Res. 2018 Jul;37(1):163. 